\title{
A discussion of the Muscle Tuning and the Preferred Movement Path concepts - comment on Nigg et al.
}

\author{
Peter Federolf ${ }^{1, *}$, Aude-Clémence M. Doix ${ }^{1}$ \& Daniel Jochum ${ }^{1}$ \\ 1 Department of Sport Science, University of Innsbruck, Innsbruck, Austria \\ * Corresponding author: Department of Sport Science, University of Innsbruck, Fürstenweg 185, A-6020 Innsbruck, Austria, \\ Tel: +43 (0) 512-507 45862 \\ Email: peter.federolf@uibk.ac.at
}

\section{COMMENTARY}

\section{Article History:}

Submitted $8^{\text {th }}$ January 2018

Accepted $9^{\text {th }}$ January 2018

Published $18^{\text {th }}$ April 2018

Handling Editor:

Markus Tilp

Karl-Franzens-University Graz, Austria

Editor-in-Chief:

Martin Kopp

University of Innsbruck, Austria

\section{ABSTRACT}

Nigg and colleagues propose two new paradigms, the Muscle Tuning and the Preferred Movement Path concepts. The purpose of this commentary is to discuss plausibility and challenges of these two concepts. Both concepts are highly plausible from a mechanical point of view and they also go in line with every-day observations. The main challenges for the muscle tuning paradigm are that (a) this mechanism is only one of several mechanisms in how the body adapts to impacts, and (b) it is very difficult to develop testable predictions from this paradigm since the mechanical (vibrational) properties of the leg are highly subject-specific and complex. The main open questions regarding the preferred movement path paradigm relate to (a) its integration with the concepts for movement variability, and (b) to the circumstances under which the preferred movement path might change.

Keywords:

Human Movement - Adaptation to Impact - Movement variability - Kinematic Analysis - Principal

Component Analysis PCA

Citation:

Federolf, P., Doix, A.-C. M. \& Jochum, D. (2018): A discussion of the Muscle Tuning and the Preferred Movement Path concepts - comment on Nigg et al. Current Issues in Sport Science, 3:103. doi: 10.15203/CISS_2018.103

This is a commentary on a CISS report article authored by Nigg, B. M., Mohr, M. \& Nigg, S. R. (2017). Muscle tuning and preferred movement path - a paradigm shift. Current Issues in Sport Science, 2:007. doi: 10.15203/CISS_2017.007

Two paradigm shifts are outlined in the target article of Nigg and colleagues published recently in CISS (Nigg, Mohr, \& Nigg, 2017) and also in some previous publications (Nigg, 2001, 2010; Nigg, Baltich, Hoerzer, \& Enders, 2015; Nigg et al., 2017). The first proposed paradigm shift suggests that the impact forces occurring as the foot strikes the ground during running should not be regarded as a major cause for running related injuries. While this suggestion at first glance seems counter-intuitive, the authors present functional considerations as well as epidemiological observations to substantiate this conclusion. Similarly, the paradigm that foot pronation is a potential cause for running injuries is challenged by conceptual considerations as well as an apparent lack of epidemiological support. Instead, the authors propose two other biomechanical mechanisms which, in their opinion, are more relevant in the context of running and running-related injuries: the "muscle tuning" and the "preferred movement path" concepts. The current commentary will discuss these two concepts from a critical perspective.

\section{Muscle tuning}

The underlying idea for the muscle tuning concept is that mechanical systems of hard and soft materials (bone and soft tissues in the leg), when exposed to impacts (such as the heel strike on the ground in running), are expected to exhibit vibrations. In fact, when watching the calf or other muscle groups of the leg during heel-toe running, one can observe a shockwave travelling through these soft tissue compartments. However, these shockwaves usually do not trigger significant vibrations. The muscle tuning concept proposed by Benno Nigg suggests that the neuromuscular system actively adapts ("tunes") the 
mechanical properties of the leg in such a way that vibrations are critically damped.

From mechanical, motor learning, or evolutionary perspectives this concept is highly plausible: When the neuromuscular system is expecting an impact, such as a heel strike in running or a jump landing, then fewer vibrations are observed in the biomechanical systems than one would expect in similar passive mechanical systems. When the system is not expecting the impact, e.g. an unexpected step when ascending or descending a stair, then much larger shockwaves and vibrations can be observed. It makes sense that an adaptation to minimize vibrations in a movement is part of the motor learning process when acquiring that movement. Additionally, considering that internal vibrations within the biomechanical system would excessively strain particularly the structures that link soft and hard tissues, it also makes sense that the skill and the physiological prerequisites for such an adaptation mechanism have developed evolutionarily.

However, there are at least two criticisms that can be brought forward. First, muscle tuning is not the only mechanism playing a role when the biomechanical system prepares for impact. Particularly the tendons play an important role, for example, in order to exploit the stretch-shortening cycle (Komi, 2000; Nicol, Avela, \& Komi, 2006) or simply to absorb and then dissipate impact energy (Roberts \& Konow, 2013). Both of these mechanisms require pre-activation of the muscles. During impacts it is also common to observe muscular co-contraction in order to stabilize the joints (Hirokawa, Solomonow, Luo, Lu, \& D'ambrosia, 1991). Hence, in actual movements "muscle tuning" most likely takes the form of a modulation of muscular preactivation, co-contraction or movement-related activation; it is most likely not an activation that can be observed or studied independently.

Second, the vibrational properties of a system depend on the material and geometric properties of that system. However, it is impossible to correctly model the actual (and constantly changing) mechanical and geometrical properties of the leg during a movement, and hence, it seems largely impossible to make anything but rough estimates about its vibrational properties. Consequently, the muscle tuning principle can serve as one of the reasons for high variability and high subject-specificity in impact responses (Huber et al., 2013), but future hypothesisdriven research is significantly hampered by the difficulty in developing precise predictions.

\section{Preferred movement path}

The preferred movement path paradigm is usually introduced from a perspective of orthotic intervention aiming at changing skeletal alignment (Nigg et al., 2017; Nigg, 2010; Nigg et al., 2017). Ample research by Benno Nigg and many other colleagues found that such interventions usually fail to produce significant changes in the joint kinematics (Nigg, 2010). Instead, it seems that the neuromuscular system prefers a spe- cific, individual kinematic pattern, called the "preferred movement path". When external interventions interfere with this "path", the neuromuscular system counteracts, e.g. with modified muscle activation, such that the original movement pattern is preserved.

As such, the preferred movement path paradigm represents a compelling synthesis of conclusions from many empirical studies. It is also a common every-day observation that human gait has a specific, highly individual pattern to it - sometimes we can identify a person only from observing their gait characteristics. However, a number of open questions and also some discrepancies in the definition of what the preferred movement path exactly is, remain. One unresolved issue is how the preferred movement path concepts can be integrated with another important concept in human movement science, the concept of movement variability. We know from Bernstein (Bernstein, 1966), Latash (Latash, 2000) and many others (Bartlett, Wheat, \& Robins, 2007) that variability is inherent to human movement. In gait, no step is exactly equal to any previous step. Is it then possible that there is one single trajectory that the neuromuscular system is trying to perform? - One solution to this question might be that the preferred movement path should really be interpreted as a "path" of similar trajectories, rather than as one single trajectory. Similar to the concepts of uncontrolled manifold hypothesis (Latash, Scholz, \& Schöner, 2002) or minimal intervention principle (Todorov \& Jordan, 2002, 2003) we could speculate, that the sensorimotor system allows variability as long as the trajectories remain within the preferred movement path, but starts to actively intervene when too large deviations are detected.

Another open question is whether the preferred movement path can change. In their target article Nigg and colleagues suggest that training, injury or fatigue may affect the preferred movement path. In our opinion, long term adaptations seem plausible, for example, a recent study found differences in movement patterns between high-mileage and low-mileage runners (Boyer, Freedman Silvernail, \& Hamill, 2014), which suggest that an adaptation in the preferred movement path seems likely. Short-term adaptations, however, seem less plausible, especially if the preferred movement path is based on a skeletal"minimal resistance path" (Wilson, Feikes, Zavatsky, Bayona, \& O'Connor, 1996). If anatomical properties shape the preferred movement path, then it seems rather unlikely that it would change due to fatigue or other short term effects. To be precise, fatigue can change running kinematics (Chan-Roper, Hunter, Myrer, Eggett, \& Seeley, 2012). However, if the preferred movement path is defined as a theoretical optimum movement pattern, then such kinematic changes due to fatigue are only a sign that the neuromuscular system is no longer able to perform close to its theoretical optimum. The observation that novice runners show greater changes with fatigue than experienced runners (Maas, De Bie, Vanfleteren, Hoogkamer, \& Vanwanseele, 2017) could be a sign that experienced runners have developed better strategies allowing them to perform close to the preferred movement path despite fatigue. 
Most studies investigating the preferred movement path focus on individual joint angles, e.g. at the ankle or knee (Nigg et al., 2017). However, within the biomechanical system in a specific movement (running), these angles cannot change independently from each other (Federolf, Boyer, \& Andriacchi, 2013). For future research into the preferred movement path we would argue that methods that study the coordination of segment movements, such as principal component analysis PCA (Federolf, Reid, Gilgien, Haugen, \& Smith, 2014; Federolf, 2016; Gløersen, Myklebust, Hallén, \& Federolf, 2018) should be applied. One reason is that PCA-based analyses tend to be more sensitive compared to many other methods, for example, while Boyer and colleagues found differences in coordination patterns between experienced and non-experienced runners (Boyer et al., 2014), other studies employing different methods did not (Floria, Sanchez-Sixto, Ferber, \& Harrison, 2018).

\section{Résumé}

The current comment on the muscle tuning and preferred movement path concepts may appear to focus on challenges, unclear aspects or discrepancies in these concepts. This is not meant as a challenge to these concepts, which, in our opinion, are plausible and convincing. Rather, the current comment is meant as an outline for future work that should be done.

\section{Funding}

The authors have no funding or support to report.

\section{Competing Interests}

The authors have declared that no competing interests exist.

\section{Data Availability Statement}

All relevant data are within the paper.

\section{References}

Bartlett, R., Wheat, J., \& Robins, M. (2007). Is movement variability important for sports biomechanists? Sports Biomechanics, 6(2), 224-243. doi:10.1080/14763140701322994

Bernstein, N. (1966). The co-ordination and regulation of movements. The co-ordination and regulation of movements.

Boyer, K. A., Freedman Silvernail, J., \& Hamill, J. (2014). The role of running mileage on coordination patterns in running. Journal of Applied Biomechanics, 30(5), 649-654. doi:10.1123/ jab.2013-0261
Chan-Roper, M., Hunter, I., Myrer, J. W., Eggett, D. L., \& Seeley, M. K. (2012). Kinematic changes during a marathon for fast and slow runners. Journal of sports science \& medicine, 11(1), 77.

Federolf, P., Boyer, K., \& Andriacchi, T. (2013). Application of principal component analysis in clinical gait research: identification of systematic differences between healthy and medial knee-osteoarthritic gait. Journal of biomechanics, 46(13), 2173-2178.

Federolf, P., Reid, R., Gilgien, M., Haugen, P., \& Smith, G. (2014). The application of principal component analysis to quantify technique in sports. Scandinavian Journal of Medicine \& Science in Sports, 24(3), 491-499.

Federolf, P. A. (2016). A novel approach to study human posture control:"Principal movements" obtained from a principal component analysis of kinematic marker data. Journal of Biomechanics, 49(3), 364-370.

Floria, P., Sanchez-Sixto, A., Ferber, R., \& Harrison, A. J. (2018). Effects of running experience on coordination and its variability in runners. Journal of Sports Sciences, 36(3), 272-278. doi:10.1080/02640414.2017.1300314

Gløersen, Ø., Myklebust, H., Hallén, J., \& Federolf, P. (2018). Technique analysis in elite athletes using principal component analysis. Journal of Sports Sciences, 36(2), 229-237.

Hirokawa, S., Solomonow, M., Luo, Z., Lu, Y., \& D’ambrosia, R. (1991). Muscular co-contraction and control of knee stability. Journal of Electromyography and Kinesiology, 1(3), 199208.

Huber, C., Federolf, P., Nüesch, C., Cattin, P. C., Friederich, N. F., \& von Tscharner, V. (2013). Heel-strike in walking: Assessment of potential sources of intra-and inter-subject variability in the activation patterns of muscles stabilizing the knee joint. Journal of Biomechanics, 46(7), 1262-1268.

Komi, P. V. (2000). Stretch-shortening cycle: a powerful model to study normal and fatigued muscle. Journal of Biomechanics, 33(10), 1197-1206.

Latash, M. (2000). There is no motor redundancy in human movements. There is motor abundance. Motor Control, 4(3), 259-261.

Latash, M. L., Scholz, J. P., \& Schöner, G. (2002). Motor control strategies revealed in the structure of motor variability. Exercise and Sport Sciences Reviews, 30(1), 26-31.

Maas, E., De Bie, J., Vanfleteren, R., Hoogkamer, W., \& Vanwanseele, B. (2017). Novice runners show greater changes in kinematics with fatigue compared with competitive runners. Sports Biomechanics, 1-11. doi:10.1080/14763141.2017.134 7193

Nicol, C., Avela, J., \& Komi, P. V. (2006). The stretch-shortening cycle. Sports Medicine, 36(11), 977-999.

Nigg, B., Mohr, M., \& Nigg, S. R. (2017). Muscle tuning and preferred movement path-a paradigm shift. Current Issues in Sport Science (CISS).

Nigg, B. M. (2001). The role of impact forces and foot pronation: a new paradigm. Clinical Journal of Sport Medicine, 11(1), 2-9. 
Nigg, B. M. (2010). Biomechanics of Sport Shoes: University of Calgary.

Nigg, B. M., Baltich, J., Hoerzer, S., \& Enders, H. (2015). Running shoes and running injuries: mythbusting and a proposal for two new paradigms:'preferred movement path'and 'comfort filter'. Britisch Journal of Sports Medicine, bjsports-2015-095054.

Nigg, B. M., Vienneau, J., Smith, A. C., Trudeau, M. B., Mohr, M., \& Nigg, S. R. (2017). The preferred movement path paradigm: influence of running shoes on joint movement. Medicine \& Science in Sports \& Exercise, 49(8), 1641-1648.

Roberts, T. J., \& Konow, N. (2013). How tendons buffer energy dissipation by muscle. Exercise and Sport Sciences Reviews, 41(4).

Todorov, E., \& Jordan, M. I. (2002). Optimal feedback control as a theory of motor coordination. Nature Neuroscience, 5(11), 1226-1235.

Todorov, E., \& Jordan, M. I. (2003). A minimal intervention principle for coordinated movement. Paper presented at the Advances in neural information processing systems.

Wilson, D., Feikes, J., Zavatsky, A., Bayona, F., \& O'Connor, J. (1996). The one degree-offreedom nature of the human knee joint-basis for a kinematic model. Paper presented at the Proceedings, Ninth Biennial Conference. 